\title{
Appraisal and discernment of prevalent drug-drug interactions in patients with psychiatric disorders
}

\author{
Axa Jacob*, Cristin Simon Thomas, Anay Deore, Prasanna Deshpande
}

Department of Clinical Pharmacy, Bharati Hospital and Research Centre, Dhankawadi, Pune, Maharashtra, India

Received: 31 May 2021

Revised: 27 June 2021

Accepted: 29 June 2021

\section{*Correspondence:}

Dr. Axa Jacob,

Email: axajacobofficial@gmail.com

Copyright: ( $)$ the author(s), publisher and licensee Medip Academy. This is an open-access article distributed under the terms of the Creative Commons Attribution Non-Commercial License, which permits unrestricted non-commercial use, distribution, and reproduction in any medium, provided the original work is properly cited.

\begin{abstract}
Background: Drug-drug interactions (DDIs) contribute majorly to hospital admissions, treatment failures, avoidable medical complications and subsequent healthcare costs. Thus, we employ a mechanistic approach to prospectively investigate the incidence of potential DDIs in the psychiatric patients in a clinical setting.

Methods: In this prospective, observational, multi centred study conducted for a span of 6 months, psychiatric inpatients ( $\geq 18$ years) prescribed with 2 or more medications daily for any medical illness were included. The secured prescriptions of the inpatients selected in accordance to the inclusion criteria were then assessed for DDIs using Micromedex ${ }^{(\mathrm{TM})}$ as a standard.

Results: Of the total 400 enrolled participants, 383 (95\%) of them showed at least one pDDI regardless of the severity. An average of 7.33 interactions per patient was also deduced. A high prevalence of pDDIs totalling to 2900 was recorded in our study with an average of 7.33 interactions per patient. Most of the interactions were of major $(56.52 \%)$ and moderate severity (39.07) followed by contraindicated (2.55) and minor (1.83). Cardiovascular system (41.77\%) had the highest potential to be affected due to the pDDIs identified. Trihexyphenidyl, haloperidol, promethazine, amisulpride, risperidone, divalproex, trifluoperazine, olanzapine and clozapine where among the most commonly encountered drugs in these interactions.

Conclusions: A high prevalence of pDDIs totalling to 2900 was recorded in our study with an average of 7.33 interactions per patient. A significant association of the pDDIs with variables such as age, gender, diagnosis and total number of drugs used was identified. More studies are required to explore the overall pattern of DDIs in psychiatric patients along with their levels and correlation with different risk factors. Careful monitoring and documentation are necessary to prevent further complications thereby improving the therapeutic outcome.
\end{abstract}

Keywords: Prevalence, DDIs, Psychotropic drugs

\section{INTRODUCTION}

\section{Psychiatric disorder}

The diagnostic and statistical manual of mental disorders, $5^{\text {th }}$ edition (DSM-V) promulgated the definition of psychiatric disorder as a behavioural or psychological syndrome or pattern that causes significant distress or impairment of personal functioning in an individual. ${ }^{1}$ The features presented by a mental illness may be persistent, worsening and remitting, or occur as a single episode.
Many disorders have been described, with signs and symptoms that vary widely between specific disorders. The causes of mental disorders are often unclear. Mental disorders are generally characterized by some combination of abnormal thoughts, emotions, behaviour and relationship with others. ${ }^{2}$ Absence of mental health is a great burden to the economic, political and social functioning of human beings, society, and nation. The major psychiatric disorders as per DSM-V, a standard language to communicate about diagnostic criteria and classification of mental disorders, are neurodevelopmental 
disorders, schizophrenia spectrum and other psychotic disorders, bipolar and related disorders, depressive disorders, anxiety disorders, obsessive-compulsive and related disorders, trauma- and stressor-related disorders, dissociative disorders, somatic symptom and related disorders, feeding and eating disorders, elimination disorders, sleep-wake disorders, sexual dysfunctions, gender dysphoria, disruptive/impulse-control/conduct disorders, substance-related and addictive disorders, neurocognitive disorders, personality disorders, paraphilic disorders, and other mental disorders. ${ }^{3}$

\section{Prevalence of psychiatric disorders}

\section{Indian scenario}

India has a lifetime mental morbidity of $13.9 \%$ with a suicidal risk of $6.4 \% .^{4}$ The prevalence of mental illness in India is 58.2 and 73 per 1000 population as reported by meta-analysis of certain epidemiological studies. ${ }^{5}$ One of the costliest mental illness in terms of both human suffering and social expenditure is schizophrenia. ${ }^{6}$ About 5-10 in 1000 people are affected with schizophrenia in India. ${ }^{7}$ In India, there is no nationwide study to evaluate the prevalence rates of BPAD. ${ }^{8} 0.5 \%$ Indians suffer from this disorder. ${ }^{2,7}$

\section{Global scenario}

According to Felker et al, psychiatric patients have more than twice the standardized mortality ratios for both natural and unnatural causes of death when compared to that of the general population. ${ }^{9}$ Schizophrenia is estimated to have a lifetime prevalence of $0.3-0.7 \%$ worldwide. ${ }^{6} \mathrm{~A}$ recent international review ${ }^{10}$ of both DSM-IV bipolar I and II disorders in population studies yielded an aggregate cross-study lifetime prevalence estimate of $1.2 \%$, ranging from $0.1 \%$ in Nigeria $^{11}$ to $3.3 \%$ in the U.S.. ${ }^{12}$ Another common mental disorder is depression and is one of the main causes of disability universally. Globally, an estimated 300 million people are affected by depression. ${ }^{2}$ Worldwide, 47.5 million people have dementia. ${ }^{2}$

\section{Psychotropic treatment ${ }^{13}$}

At present, mental disorders are managed by psychological or biological treatment. Approaches in psychological therapies include cognitive therapy, behavioural therapy, family focused therapy or psychoanalysis. Biological therapy for mental disorders generally involves the use of some form of physical intervention (such as pharmacotherapy). The medicines with specific abilities to produce effects upon emotion and behaviour and which are most commonly used in the management of mental disorders are often referred to as psychotropic drugs. This area of pharmacology is also known as psychopharmacology.

\section{Drug-drug interactions (DDIs)}

A DDIs is defined as the pharmacological or clinical response to the administration or co-exposure of a drug with another drug that modifies the patient's response to the drug index. ${ }^{14}$ DDIs can lead to alteration of therapeutic response or increase untoward effects of many drugs. ${ }^{15} \mathrm{Old}$ age, taking increased number of medications, long hospital stays, gender and co morbid conditions have been reported as common risk factors for DDIs. ${ }^{16}$ As most psychotropic medications are metabolized by the cytochrome (CYP) enzyme system, it may be predicted that the risk of DDIs will increase as a result of polypharmacy. ${ }^{17}$ The issue of DDIs needs more attention in the case of hospitalized patients due to severity of disease, polypharmacy, comorbid conditions, chronic diseases, complex therapeutic regime, and frequent modification in therapy. ${ }^{15}$ Drug interactions between the drugs vary with changes in their underlying mechanism. Drug interactions are categorized into behavioural, pharmaceutical, pharmacokinetic, and pharmacodynamic DDIs. ${ }^{18}$

\section{DDIs in psychiatry}

The breakthrough of new psychopharmacologic agents facilitated the availability of more therapeutic options but has also complicated the patient treatment. The likelihood of drug interactions in psychiatry is attributed to combination therapy which culminates as an increased risk of adverse outcomes to the patients. ${ }^{19}$ There exists a variation in the significance of a drug interaction between individuals depending on factors like co-morbidities, gender, and age. ${ }^{20}$ Psychiatric medications can account for up to $50 \%$ of the ADRs in hospitalized psychiatric patients, multiple of which can be attributed to DDIs as revealed by a recently published study. ${ }^{21}$ Guo et al have reviewed the medical records of health insurance system and detected potentially dangerous drug interactions in approximately $23 \%$ of patients taking antipsychotic medications. ${ }^{22}$ Another study revealed that DDIs account for an estimated $26 \%$ of ADRs requiring hospital admissions whereas prevalence of DDIs in psychiatric hospital settings has been estimated in some studies to be in the range of 27.8 to $51.4 \% .^{23,24}$ Hence it is important to contemplate potentially hazardous interactions in psychiatry.

\section{Prevalence of psychiatric DDIs}

\section{Global and Indian scenario}

An elevation in the elderly population world-wide has led to concerns regarding the burden of DDI-related ADRs. Attributing to the specific characteristics of the elderly such as physiologic modifications related to ageing processes, the prevalence of pDDIs is elevated in the elderly and ranges from 42.5 to $54.4 \% .{ }^{25}$ In a prospective study conducted in Taiwan, a total of 130 potential interactions were detected in $339(63.1 \%)$ of the 537 medication profiles. $^{26}$ Lima and De Bortoli Casiani 
demonstrated that incidence of DDIs increases by $10-20 \%$ in patients using 10-20 drugs. ${ }^{27}$

A recent study from India on the incidence and predictors of adverse drug reactions caused by DDIs in psychiatric patients was found to be $12 \%$. Pharmacodynamic interactions accounted for the majority $(68.5 \%)$ of ADRs. The greatest propensity to interact with other medications was exhibited by risperidone with 41 occurrences. ${ }^{28}$

In this study, the authors aimed to assess commonly occurring pDDIs, the psychiatric diagnosis of patients in the study, manifestation of comorbidities, customary psychotropic use, prevalence of pDDIs and their classification based on severity/documentation/onset, adverse clinical outcomes of pDDIs, and common interacting drug combinations with their probable mechanism of interaction along with their clinical management. This observational study also determines the statistical association of parameters like age, gender, comorbidity, diagnosis, body system, onset, documentation, psychotropic and non-psychotropic drug use against different severities of pDDIs.

\section{METHODS}

This prospective, observational, multi centered study was of 6 months duration conducted from October 2017 to March 2018 on patients under inclusion criteria. The four study sites were: site I -Bharati hospital and research center, Dhankawadi, Pune-411043; site II-Chaitanya institute for mental health, Bhagat Puram, Khadi Machine Chowk, Pune- 411048; site III-Chaitanya Institute for Mental Health, Behind Wonder City, Katraj, Pune411046; site IV-Chaitanya institute for mental health, popular prestige commercial complex, Warje, Pune411052. Psychiatric inpatients ( $\geq 18$ years) prescribed with 2 or more medications daily for any medical illness were included in the study. The exclusion criteria were medicolegal cases of any kind and interactions of herbal/topical medications.

We determined the recommended sample size using Raosoft sample size calculator. ${ }^{31}$ Considering parameters like margin of error, confidence level, population size, response distribution as 5\%, 95\%, 20000, 50\% respectively, our recommended sample size was 377 . However, we proceeded with 400 as our final sample size.

Micromedex ${ }^{(\mathrm{TM})}$, an online evidence-based database was used for identifying DDIs in the study. It includes "in-line" referenced information about drugs, toxicology, diseases, acute care, and alternative medicine for healthcare professionals to make informed clinical diagnosis and treatment decisions. It is a source of quick and reliable drug information.

Ethical approval for the study was obtained from the ethics committee of Bharati medical college and Chaitanya institute for mental health. Inpatients were selected in accordance to the inclusion criteria. Detailed information regarding the study objectives were explained to the patients and consent was taken thereby ascertaining their willingness to participate in the said study. Prescriptions were procured for demographic details (e.g., patient' $s$ name, age, sex, date of admission), confirmed diagnosis, current medication (with brand and generic name, date started and stopped). The required details were noted in the self-predesigned patient profile form. The prescriptions were assessed for DDIs using Micromedex $^{(\mathrm{TM})}$ as a standard. During assessment of drug interactions, the DDIs were assembled depending on the parameters defined by Micromedex ${ }^{(\mathrm{TM})}$. The classifications made to assess drug interactions and to formulate the results were according to severity (Contraindicated/major/moderate/minor/ unknown), effect on body system (Cardiovascular/ neurologic/ANS/hematologic/endocrine and metabolic/ respiratory/others), onset (Not specified/ Rapid/Delayed), documentation (Excellent/ good/fair/unknown), probable mechanism (QT prolongation/CNS depression/delayed gastric emptying, etc.), and clinical management (Monitor ECG/separate administration of two interacting drugs by at least 2 hours/monitor glucose levels/monitor for signs of toxicity, etc.).

The severity of drug interactions as defined by Micromedex ${ }^{(\mathrm{TM})}$ were contraindicated (the drugs are contraindicated for concurrent use), major (the interaction may be life threatening and/or require medical interventions to minimise or prevent serious adverse effects), moderate (the interaction may result in exacerbation of the patient's condition and/or require alternate therapy), minor (the interaction would have limited clinical effects. Manifestations may include an increase in the frequency or severity of the side effects but generally would not require a Major alteration in therapy), and unknown (Unknown). ${ }^{32}$

The chi-square test was used to establish the association between the categorical variables. $\mathrm{P}$ values were obtained from the Chi square calculator. ${ }^{37}$ The associations were established against contraindicated drug interactions (Dis), major DIs, moderate DIs, minor DIs, unknown DIs and total number of DIs. Parameters which were considered for association are age, gender, presence and absence of comorbidity, psychiatric diagnosis, clinical outcome of DIs i.e., the body system affected, onset of DIs, documentation of DIs, psychotropic, and nonpsychotropic drugs administered to patient.

\section{RESULTS}

\section{General patient characteristics}

For the sample size of 400 , the margin of error was found to be $4.85 \%$ according to Raosoft sample size calculator considering parameters like confidence level, population size, response distribution as 95\%, 20000, 50\% respectively. This study consisting of a total number of 400 patients, had $252(63 \%)$ male and $148(37 \%)$ female [male 
to female ratio being 252:148] participants with a mean age of $46.24 \pm 16.81$ years. Around 161 patients diagnosed with psychiatric disease belong to the age range of 31 to 50 years accounting up to $40.25 \%$ of the patients under study (Table 1).

Table 1: Demographic profile of the study participants.

\begin{tabular}{|c|c|c|}
\hline Demographic profile & Frequency & Percent $(\%)$ \\
\hline \multicolumn{3}{|l|}{ Age range (years) } \\
\hline 10 to 20 & 18 & 4.5 \\
\hline $21-30$ & 68 & 17 \\
\hline $31-40$ & 76 & 19 \\
\hline $41-50$ & 85 & 21.25 \\
\hline $51-60$ & 65 & 16.25 \\
\hline $61-70$ & 50 & 12.5 \\
\hline $71-80$ & 28 & 7 \\
\hline $81-90$ & 8 & 2 \\
\hline $91-100$ & 2 & 0.5 \\
\hline \multicolumn{3}{|l|}{ Sex } \\
\hline Male & 252 & 63 \\
\hline Female & 148 & 37 \\
\hline
\end{tabular}

In the total 3375 drugs prescribed, $2053(60.83 \%)$ were psychotropic whereas $1322(39.17 \%)$ were given for underlying co morbid conditions. An average of 8.44 medications per patient was also identified. The number of orally administered drugs were 3117 (92.36\%), 222 $(6.58 \%)$ administered intramuscularly, 33 (0.68\%) intravenously and $3(0.09 \%)$ subcutaneously. Amongst the registered patients, the most prevalent diagnosis was found to be schizophrenia with a total of 225 patients followed by mental retardation with psychosis (38) and bipolar disorder (31) (Table 2). This study also analysed patients suffering from various co-morbid conditions for which they were being prescribed medications along with antipsychotics. Such patients came to a total count of 284 (71\%) (Figure 1). The study provides information regarding the most commonly used psychotropics by patients from all the 4 four sites. The highly used antipsychotic was discovered to be trihexyphenidyl (9.8\%) with the least being quetiapine (2.22\%) (Figure 2).

Table 2: Psychiatric diagnosis of patients in the study.

\begin{tabular}{|lll|}
\hline Psychiatric diagnosis & Frequency & Percent $(\%)$ \\
\hline Schizophrenia & 225 & 56.25 \\
\hline Bipolar disorder & 31 & 7.75 \\
\hline $\begin{array}{l}\text { Alcoholic dependence } \\
\text { syndrome }\end{array}$ & 30 & 7.5 \\
\hline Dementia & 17 & 4.25 \\
\hline MR + psychosis & 38 & 9.5 \\
\hline Depression & 12 & 3 \\
\hline Substance Induced & 20 & 5 \\
\hline Others & 27 & 6.75 \\
\hline
\end{tabular}

MR: Mental Retardation, Others: Serotonin Syndrome (8); Increased exposure to CYP2D6 substrate (5); Potential toxicity like sedation (2), confusion (3), cardiac arrhythmias (1), orthostatic hypotension (1), hyperthermia (5), extrapyramidal effects (2).

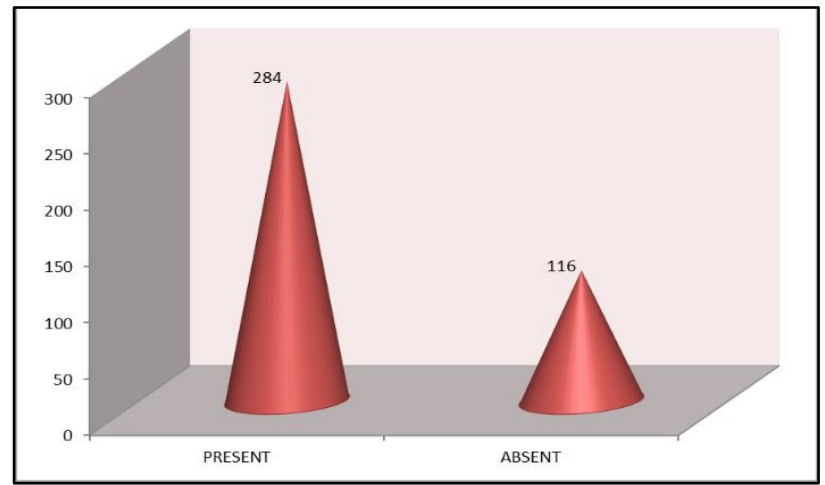

Figure 1: Manifestation of comorbidities in the patients under study.

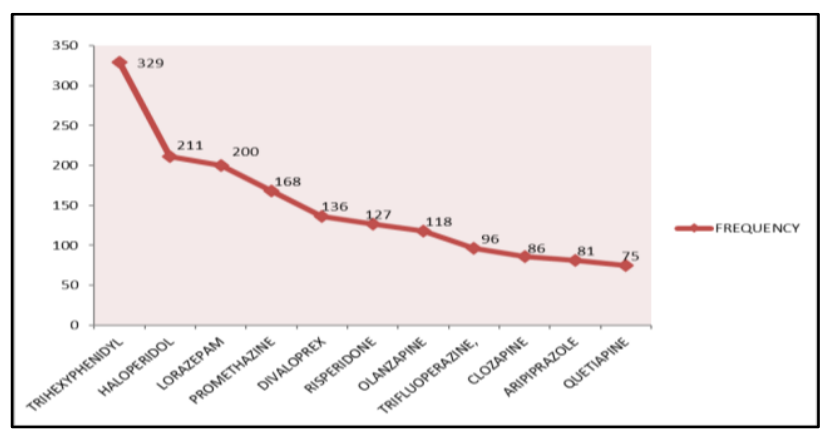

Figure 2: Customary psychotropic use in the enrolled patients.

\section{Prevalence of pDDIs}

Of the total 400 enrolled participants, $383(95 \%)$ of them showed at least one pDDI regardless of the severity. An average of 7.33 interactions per patient was also deduced.

\section{Levels of pDDIs}

Based on the onset, severity and scientific evidence, the identified pDDIs were catalogued into different levels. Amidst the total 2900 pDDIs determined, most belonged to the major (1639) and moderate (1133) severities followed by contraindicated (74) and minor (53) severities. The highest number of pDDIs was found to be in the major severity $(56.52 \%)$ of a majority had a fair documentation with onsets not specified. Appraisal of the scientific evidence revealed a total of 137 pDDIs with an excellent documentation, 750 with good and a maximum of 2013 with fair documentation. Similarly, on assessing the onset of the pDDIs, (219) were with rapid onset, (862) delayed onset and (1819) were with non-specified onset (Table 3).

\section{Effect of pDDIs on the body system}

Upon evaluation, it was found that the cardiovascular system $(41.77 \%)$ had the highest potential to be affected 
due to the pDDIs identified. A systematic review of the said parameters is reflected in Table 4.

\section{Common interacting drug combinations}

The common interacting drug combinations of each severity along with their frequencies are given in Table 5. Trihexyphenidyl, haloperidol, promethazine, amisulpride, risperidone, divalproex, trifluoperazine, olanzapine, and clozapine where among the most commonly encountered drugs in these interactions.

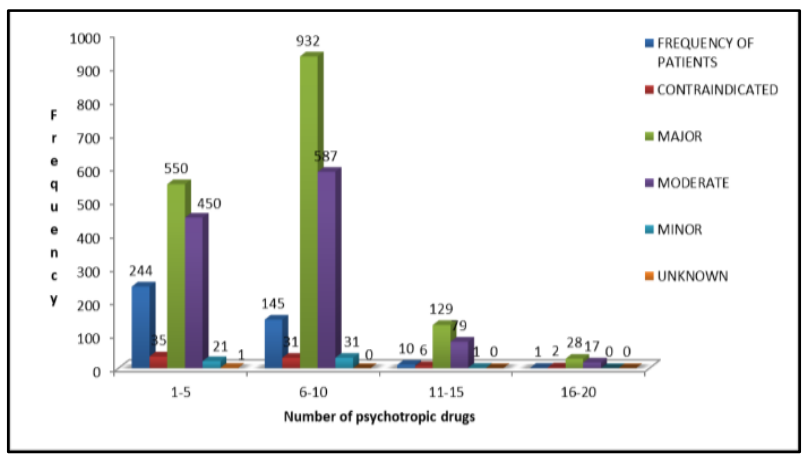

Figure 3: DIs seen with the use of psychotropic medicines by patients diagnosed with psychiatric disorders.

Table 3: Classification of identified pDDIs.

\begin{tabular}{|c|c|c|}
\hline Level & Frequency, $(n=400)$ & Percent out of total DIS (\%) \\
\hline \multicolumn{3}{|l|}{ Severity } \\
\hline Contraindicated & 74 & 2.55 \\
\hline Major & 1639 & 56.52 \\
\hline Moderate & 1133 & 39.07 \\
\hline Minor & 53 & 1.83 \\
\hline Unknown & 1 & 0.03 \\
\hline \multicolumn{3}{|l|}{ Documentation } \\
\hline Excellent & 137 & 4.72 \\
\hline Contraindicated & 0 & 0 \\
\hline Major & 47 & 1.62 \\
\hline Moderate & 86 & 2.96 \\
\hline Minor & 4 & 0.13 \\
\hline Unknown & 0 & 0 \\
\hline Good & 750 & 25.86 \\
\hline Contraindicated & 4 & 0.13 \\
\hline Major & 191 & 6.58 \\
\hline Moderate & 538 & 18.55 \\
\hline Minor & 16 & 0.55 \\
\hline Unknown & 1 & 0.03 \\
\hline Fair & 2013 & 69.41 \\
\hline Contraindicated & 70 & 2.41 \\
\hline Major & 1401 & 48.31 \\
\hline Moderate & 509 & 17.55 \\
\hline Minor & 33 & 1.13 \\
\hline Unknown & 0 & 0 \\
\hline \multicolumn{3}{|l|}{ Onset } \\
\hline Rapid & 219 & 7.55 \\
\hline Contraindicated & 4 & 0.13 \\
\hline Major & 35 & 1.20 \\
\hline Moderate & 152 & 5.24 \\
\hline
\end{tabular}

Continued. 


\begin{tabular}{|lll|}
\hline Level & Frequency, $(\mathbf{n}=\mathbf{4 0 0})$ & Percent out of total DIS $(\%)$ \\
\hline Minor & 27 & 0.93 \\
\hline Unknown & 1 & 0.03 \\
\hline Delayed & 862 & 29.72 \\
\hline Contraindicated & 0 & 0 \\
\hline Major & 97 & 3.34 \\
\hline Moderate & 745 & 25.68 \\
\hline Minor & 20 & 0.68 \\
\hline Unknown & 0 & 0 \\
\hline Not specified & 1819 & 62.72 \\
\hline Contraindicated & 70 & 2.41 \\
\hline Major & 1507 & 51.96 \\
\hline Moderate & 236 & 8.13 \\
\hline Minor & 6 & 0.20 \\
\hline Unknown & 0 & 0 \\
\hline
\end{tabular}

Table 4: Adverse clinical outcomes of pDDIs in the enrolled patients.

\begin{tabular}{|c|c|c|}
\hline Body system effect & Frequency $(\mathrm{n=3184})$ & Percentage (\%) \\
\hline Cardiovascular & 1330 & 41.77 \\
\hline Contraindicated & 72 & 2.26 \\
\hline Major & 1209 & 37.97 \\
\hline Moderate & 46 & 1.44 \\
\hline Minor & 3 & 0.09 \\
\hline Unknown & 0 & 0 \\
\hline Decreased plasma concentration & 497 & 15.61 \\
\hline Contraindicated & 0 & 0 \\
\hline Major & 26 & 0.81 \\
\hline Moderate & 449 & 14.10 \\
\hline Minor & 22 & 0.69 \\
\hline Unknown & 0 & 0 \\
\hline Increased plasma concentration & 326 & 10.23 \\
\hline Contraindicated & 0 & 0 \\
\hline Major & 66 & 2.07 \\
\hline Moderate & 254 & 7.97 \\
\hline Minor & 6 & 0.188 \\
\hline Unknown & 0 & 0 \\
\hline Autonomic nervous system & 319 & 10.01 \\
\hline Contraindicated & 0 & 0 \\
\hline Major & 73 & 2.29 \\
\hline Moderate & 246 & 7.72 \\
\hline Minor & 0 & 0 \\
\hline Unknown & 0 & 0 \\
\hline Neurological & 280 & 8.79 \\
\hline Contraindicated & 1 & 0.031 \\
\hline Major & 166 & 5.21 \\
\hline Moderate & 91 & 2.85 \\
\hline Minor & 21 & 0.659 \\
\hline Unknown & 1 & 0.031 \\
\hline Hematologic & 167 & 5.24 \\
\hline Contraindicated & 0 & 0 \\
\hline Major & 114 & 3.58 \\
\hline Moderate & 51 & 1.60 \\
\hline Minor & 2 & 0.062 \\
\hline Unknown & 0 & 0 \\
\hline Endocrine & 70 & 2.19 \\
\hline Contraindicated & 0 & 0 \\
\hline Major & 29 & 0.91 \\
\hline Moderate & 41 & 1.028 \\
\hline
\end{tabular}




\begin{tabular}{|lll|}
\hline Body system effect & Frequency $(\mathbf{n}=\mathbf{3 1 8 4})$ & Percentage $\mathbf{( \% )}$ \\
\hline Minor & 0 & 0 \\
\hline Unknown & 0 & 0 \\
\hline Musculoskeletal & 10 & 0.31 \\
\hline Contraindicated & 0 & 0.21 \\
\hline Major & 7 & 0.062 \\
\hline Moderate & 2 & 0.031 \\
\hline Minor & 1 & 0 \\
\hline Unknown & 0 & 1.50 \\
\hline Metabolic & 48 & 0 \\
\hline Contraindicated & 0 & 0.78 \\
\hline Major & 25 & 0.72 \\
\hline Moderate & 23 & 0 \\
\hline Minor & 0 & 0 \\
\hline Unknown & 0 & 1.13 \\
\hline Respiratory & 36 & 0 \\
\hline Contraindicated & 0 & 1.09 \\
\hline Major & 35 & 0.031 \\
\hline Moderate & 1 & 0 \\
\hline Minor & 0 & 0 \\
\hline Unknown & 0 & 0.031 \\
\hline Gastrointestinal & 1 & 0.031 \\
\hline Contraindicated & 1 & 0 \\
\hline Major & 0 & 0 \\
\hline Moderate & 0 & 0 \\
\hline Minor & 0 & 0 \\
\hline Unknown & 0 & 3.14 \\
\hline Other & 100 & 0.062 \\
\hline Contraindicated & 2 & 1.63 \\
\hline Major & 52 & 1.44 \\
\hline Moderate & 46 & 0 \\
\hline Minor & 0 & 0 \\
\hline Unknown & 0 & \\
\hline & & 0.09 \\
\hline
\end{tabular}

Table 5: Top 5 interacting drug combinations of each severity.

\begin{tabular}{|c|c|c|c|c|c|c|}
\hline Variables & $\mathbf{N}$ & $\begin{array}{l}\text { Effect on } \\
\text { body system }\end{array}$ & Onset & Documentation & $\begin{array}{l}\text { Probable } \\
\text { mechanism }\end{array}$ & $\begin{array}{l}\text { Clinical } \\
\text { management }\end{array}$ \\
\hline \multicolumn{7}{|c|}{ Contraindicated, $(n=74)$} \\
\hline $\begin{array}{l}\text { Amisulpride } \mathrm{x} \\
\text { Clozapine }\end{array}$ & $15(20.27)$ & Cardiovascular & $\begin{array}{l}\text { Not } \\
\text { Specified }\end{array}$ & Fair & $\begin{array}{l}\text { Additive QT } \\
\text { prolongation }\end{array}$ & Contraindicated \\
\hline $\begin{array}{l}\text { Amisulpride } \mathrm{x} \\
\text { Promethazine }\end{array}$ & $9(12.16)$ & Cardiovascular & $\begin{array}{l}\text { Not } \\
\text { Specified }\end{array}$ & Fair & $\begin{array}{l}\text { Additive QT } \\
\text { prolongation }\end{array}$ & Contraindicated \\
\hline $\begin{array}{l}\text { Amisulpride x } \\
\text { Quetiapine }\end{array}$ & $6(8.11)$ & Cardiovascular & $\begin{array}{l}\text { Not } \\
\text { Specified }\end{array}$ & Fair & $\begin{array}{l}\text { Additive QT } \\
\text { prolongation }\end{array}$ & Contraindicated \\
\hline $\begin{array}{l}\text { Amisulpride } \mathrm{x} \\
\text { Olanzapine }\end{array}$ & $6(8.11)$ & Cardiovascular & $\begin{array}{l}\text { Not } \\
\text { Specified }\end{array}$ & Fair & $\begin{array}{l}\text { Additive QT } \\
\text { prolongation }\end{array}$ & Contraindicated \\
\hline $\begin{array}{l}\text { Amisulpride } \mathrm{x} \\
\text { Apipiprazole }\end{array}$ & $6(8.11)$ & Cardiovascular & $\begin{array}{l}\text { Not } \\
\text { Specified }\end{array}$ & Fair & $\begin{array}{l}\text { Additive QT } \\
\text { prolongation }\end{array}$ & Contraindicated \\
\hline \multicolumn{7}{|l|}{ Major, $(n=1639)$} \\
\hline $\begin{array}{l}\text { Haloperidol x } \\
\text { Promethazine }\end{array}$ & $82(5.00)$ & Cardiovascular & $\begin{array}{l}\text { Not } \\
\text { Specified }\end{array}$ & Fair & $\begin{array}{l}\text { Additive effects } \\
\text { on QT interval }\end{array}$ & Monitor ECG \\
\hline $\begin{array}{l}\text { Haloperidol x } \\
\text { Risperidone }\end{array}$ & $45(2.75)$ & Cardiovascular & $\begin{array}{l}\text { Not } \\
\text { Specified }\end{array}$ & Fair & $\begin{array}{l}\text { Additive QT } \\
\text { interval } \\
\text { prolongation }\end{array}$ & $\begin{array}{l}\text { Avoid } \\
\text { concomitant use }\end{array}$ \\
\hline $\begin{array}{l}\text { Haloperidol x } \\
\text { Trifluoperazine }\end{array}$ & $40(2.44)$ & Cardiovascular & $\begin{array}{l}\text { Not } \\
\text { Specified }\end{array}$ & Fair & $\begin{array}{l}\text { Additive QT } \\
\text { prolongation }\end{array}$ & $\begin{array}{l}\text { Avoid } \\
\text { concomitant use }\end{array}$ \\
\hline $\begin{array}{l}\text { Lorazepam x } \\
\text { Olanzapine }\end{array}$ & $38(2.32)$ & $\begin{array}{l}\text { Cardiovascular/ } \\
\text { Respiratory }\end{array}$ & $\begin{array}{l}\text { Not } \\
\text { Specified }\end{array}$ & Fair & $\begin{array}{l}\text { Additive CNS } \\
\text { depression }\end{array}$ & $\begin{array}{l}\text { Avoid } \\
\text { concomitant use }\end{array}$ \\
\hline
\end{tabular}

Continued. 


\begin{tabular}{|c|c|c|c|c|c|c|}
\hline Variables & $\mathbf{N}$ & $\begin{array}{l}\text { Effect on } \\
\text { body system }\end{array}$ & Onset & Documentation & $\begin{array}{l}\text { Probable } \\
\text { mechanism }\end{array}$ & $\begin{array}{l}\text { Clinical } \\
\text { management }\end{array}$ \\
\hline $\begin{array}{l}\text { Pomethazine } \mathrm{x} \\
\text { Haloperidol }\end{array}$ & $38(2.32)$ & Cardiovascular & $\begin{array}{l}\text { Not } \\
\text { Specified }\end{array}$ & Fair & $\begin{array}{l}\text { Additive effects } \\
\text { on QT interval }\end{array}$ & Monitor ECG \\
\hline \multicolumn{7}{|c|}{ Moderate, $(n=1133)$} \\
\hline $\begin{array}{l}\text { Trihexyphenidyl } \\
\text { X Haloperidol }\end{array}$ & $\begin{array}{l}117 \\
(10.33)\end{array}$ & ANS & Delayed & Good & $\begin{array}{l}\text { Additive anti- } \\
\text { cholinergic } \\
\text { effects }\end{array}$ & $\begin{array}{l}\text { Dosage } \\
\text { adjustments } \\
\text { required }\end{array}$ \\
\hline $\begin{array}{l}\text { Trihexyphenidyl } \\
\text { X Promethazine }\end{array}$ & $103(9.09)$ & ANS & Delayed & Fair & $\begin{array}{l}\text { Delayed gastric } \\
\text { emptying }\end{array}$ & $\begin{array}{l}\text { Anticholinergic } \\
\text { use to be re- } \\
\text { evaluated every } \\
3 \text { months }\end{array}$ \\
\hline $\begin{array}{l}\text { Trihexyphenidyl } \\
\text { x Divalproex }\end{array}$ & 99 (8.74) & $\begin{array}{l}\text { Decreased } \\
\text { Plasma } \\
\text { Concentration }\end{array}$ & Delayed & Fair & Unknown & $\begin{array}{l}\text { Dose adjustment } \\
\text { for valproate } \\
\text { sodium }\end{array}$ \\
\hline $\begin{array}{l}\text { Trihexyphenidyl } \\
\mathrm{x} \\
\text { Trifluoperazine }\end{array}$ & $97(8.56)$ & ANS & Delayed & Fair & $\begin{array}{l}\text { Delayed gastric } \\
\text { emptying }\end{array}$ & $\begin{array}{l}\text { Anticholinergic } \\
\text { use to be } \\
\text { re-evaluated } \\
\text { every } 3 \text { months }\end{array}$ \\
\hline $\begin{array}{l}\text { Divaloprex x } \\
\text { Lorazepam }\end{array}$ & $67(5.91)$ & $\begin{array}{l}\text { Increased } \\
\text { Serum } \\
\text { Concentrations }\end{array}$ & Rapid & Good & $\begin{array}{l}\text { Decreased } \\
\text { lorazepam } \\
\text { metabolism }\end{array}$ & $\begin{array}{l}\text { Monitoring } \\
\text { required }\end{array}$ \\
\hline \multicolumn{7}{|l|}{ Minor, $(n=53)$} \\
\hline $\begin{array}{l}\text { Clozapine } \mathrm{x} \\
\text { Lorazepam }\end{array}$ & $19(35.85)$ & Neurologic & Rapid & Fair & Additive & $\begin{array}{l}\text { Reduce or } \\
\text { eliminate } \\
\text { caffeine } \\
\text { exposure }\end{array}$ \\
\hline $\begin{array}{l}\text { Calcium } \\
\text { Chloride } x \\
\text { Ferrous } \\
\text { Fumarate }\end{array}$ & $4(7.55)$ & $\begin{array}{l}\text { Decreased } \\
\text { Plasma } \\
\text { Concentration }\end{array}$ & Delayed & Fair & $\begin{array}{l}\text { Decreased iron } \\
\text { absorption }\end{array}$ & $\begin{array}{l}\text { Iron salts } \\
\text { should be } \\
\text { taken } 1 \mathrm{hr} \\
\text { before/after } \\
\text { calcium }\end{array}$ \\
\hline $\begin{array}{l}\text { Aspirin } x \\
\text { Ranitidine }\end{array}$ & $4(7.55)$ & $\begin{array}{l}\text { Increased/ } \\
\text { decreased } \\
\text { plasma } \\
\text { concentration }\end{array}$ & $\begin{array}{l}\text { Not } \\
\text { Specified }\end{array}$ & Excellent & $\begin{array}{l}\text { Reduced } \\
\text { absorption of } \\
\text { iron }\end{array}$ & $\begin{array}{l}\text { Coadministration } \\
\text { with caution }\end{array}$ \\
\hline $\begin{array}{l}\text { Lorazepam x } \\
\text { Caffeine }\end{array}$ & $4(7.55)$ & $\begin{array}{l}\text { Decreased } \\
\text { Plasma } \\
\text { Concentration }\end{array}$ & Rapid & Good & $\begin{array}{l}\text { CNS } \\
\text { antagonistic } \\
\text { effects }\end{array}$ & $\begin{array}{l}\text { Reduce/eliminate } \\
\text { caffeine } \\
\text { exposure }\end{array}$ \\
\hline $\begin{array}{l}\text { Ascorbic acid x } \\
\text { Cyanocobalamin }\end{array}$ & $2(3.77)$ & $\begin{array}{l}\text { Decreased } \\
\text { Plasma } \\
\text { Concentration }\end{array}$ & Delayed & Good & Unknown & $\begin{array}{l}\text { Separate } \\
\text { Administration } \\
\text { by at-least } 2 \mathrm{~h}\end{array}$ \\
\hline \multicolumn{7}{|l|}{ Unknown, $(n=1)$} \\
\hline $\begin{array}{l}\text { Lorazepam x } \\
\text { Caffeine }\end{array}$ & $1(100)$ & Neurologic & Rapid & Good & $\begin{array}{l}\text { CNS-anta- } \\
\text { gonistic effect }\end{array}$ & $\begin{array}{l}\text { Reduce caffeine } \\
\text { exposure }\end{array}$ \\
\hline
\end{tabular}

Table 6: Association of parameters of different pDDIs.

\begin{tabular}{|llllllll|} 
Parameters & $\begin{array}{l}\text { Contra } \\
\text { indicated }\end{array}$ & Major & Moderate & Minor & Unknown & $\begin{array}{l}\text { Total no. } \\
\text { of DIs }\end{array}$ & $\begin{array}{l}\text { Total no. of } \\
\text { drugs }\end{array}$ \\
\hline Age (Years) & 0.01942 & 0.4625 & 0.5166 & 0.4588 & 0.7763 & 0.8403 & - \\
\hline Gender & 0.001444 & 0.001676 & 0.060666 & 0.006035 & 0.1929 & 0.002854 & 0.140013 \\
\hline Comorbidity & 0.40827 & 0.00685 & 0.00004 & 0.006403 & 0.1188 & 0.000562 & 0.00133 \\
\hline Diagnosis & 0.009494 & 0.000005414 & 0.001006 & 0.2944 & 0.116 & 0.00006919 & 0.00152 \\
\hline Body system & 0 & 0 & 0 & 0 & 0.5003 & 1 & - \\
\hline ++++Onset & $<0.00001$ & $<0.00001$ & $<0.00001$ & $<0.00001$ & 0.002253 & 1 & - \\
\hline Documentation & 0.00001722 & $<0.00001$ & $<0.00001$ & $<0.00001$ & 0.2389 & 0.877 & - \\
\hline Psychotropic & 0.002546 & 0 & 0 & 0.02017 & 0.8876 & 0 & 0.00001109 \\
\hline $\begin{array}{l}\text { Non- } \\
\text { psychotropic }\end{array}$ & 0.1254 & 0.0008917 & 0.001671 & 0 & 0.8791 & 0.0002478 & 0.000003155 \\
\hline
\end{tabular}




\section{DISCUSSION}

Based on our estimation, a comprehensive assessment of the identification of DDIs selectively in psychiatric pharmacotherapy is very rare in Indian settings. Our study strived to bridge this deficit by employing a mechanistic approach by prospectively investigating the incidence of potential DDIs in the psychiatric patients in a clinical setting.

In Pakistan, Ismail et al had conducted a similar study enrolling a total of 415 patients. ${ }^{24}$ Mezgebe et al, who carried out the study in Ethiopia, however just had 216 participants. ${ }^{33}$ Our study consisted a total of 400 patients. Age wise, we saw a median of 45 years which was different from others (25-27 years). Based on the male female ratio, both the above-mentioned studies almost had an equal proportion (male (47-54\%): female (47-53\%). ${ }^{24,33}$ However in our study, we had a greater percentage of males enrolled (63:37\%). Diagnosis wise, Mezgebe et al had reported that patients with schizophrenia was seen the most $(57 \%) .{ }^{33}$ Coincidently, the same case was also seen in our study $(56.25 \%)$.

Like us, most of the studies involved with a similar objective used Micromedex as their standard. ${ }^{24,28,34,38}$. Sengul et al though, used Drugs.com as their standard for reference. ${ }^{35}$ While considering the total number of pDDIs seen per study, as of yet Mezgebe et al had reported a greater number with $81.8 \%$ of their patients suffering from at least one pDDI regardless of the severity. ${ }^{33}$ In comparison, Ismail et al and Lucca et al had reported a relatively lower percentage $(64.8 \% ; 55.2 \%) .{ }^{24,28}$ During the duration of our study however, we had discovered an exorbitant amount of pDDIs accounting up to $95 \%$.

Assessment of severity is essential to qualify the medical risk of the interaction. ${ }^{36}$ All the studies showed a lower proportion of pDDIs with "contraindicated" severity (0.5$3 \%) .{ }^{24,28,33}$ Our study also had the same inclination. Based on the "major" severity, the mentioned studies showed results in the range of $15-43 \% .^{24,28,33-35}$ In contrast, we determined a higher level of the said severity (56.52\%). Furthermore, we also saw a lower level of those with "moderate" severity (39.07\%) than the others (50$87 \%) .{ }^{24,33-35}$ Only Lucca et al had identified a similar level as us. When analyzing the "minor" severity, we saw that Lucca et al showed a rate of $34.82 \% .^{28}$ This was higher than what was seen with the others $(0.2 \%-5 \%){ }^{24,33-35}$ Nevertheless, the amount of "minor" pDDIs in our dissertation fell in the range that was seen in majority of the studies $(1.83 \%)$. Most of the studies produced the maximum number of pDDIs in "moderate" severity (50$90 \%) .{ }^{24,28,33-35}$ Whereas, ours produced the highest number with "major" severity $(56.52 \%)$; others being of the range $15-43 \% .^{24,28,33-35}$

Onset of action of the pDDIs is another essential parameter as it indicates how quickly the interaction may occur. ${ }^{36}$ In previous studies, the reported pDDIs with "rapid" onset generally fell in the range of 3-24\%.$^{24,28,33,34}$ Similarly, so did our study (7. 55\%). However, our study also reported a decreased level pDDIs with "delayed" onset (29.72\%) than others (50-81\%). ${ }^{24,28,33,34}$ This is mainly because we had a higher level of pDDIs with "not specified" documentation than them $(62.72 \%)$. $^{33,34}$

Documentation of a pDDI detects the quality and quantity of the medical literature supporting the inclusion in the data. ${ }^{36}$ Ismail et al reported $4.6 \%$ of their pDDIs with excellent documentation. ${ }^{24}$ Even we recorded a similar level $(4.72 \%)$. Their percentage of pDDIs with "good" documentation was higher than those with "fair" documentation $(66.4 \%>29 \%) .{ }^{24,33}$ Our study though, produced the opposite result [fair $(69.41 \%)>$ good $(25.86 \%)]$.

The comparison of the clinical outcomes determined in our dissertation was done in relation to Sengul et al. ${ }^{35}$ The study detailed a higher risk of ANS related clinical outcomes $(38.6 \%)$ of all drug interactions. In contrast, we recorded a lower level at $9.84 \%$. They even detailed an elevated level of interaction affecting the respiratory $(24.5 \%)$ and hematological system $(11.9 \%)$ than us (1.11\%: 5.15\%). However, our study reported a higher number of interactions affecting the cardiovascular system $(41.01 \%)$ than them $(14.6 \%) .{ }^{35}$

We discovered that the highest number of pDDIs occurred in the age group between 40-51 years totalling up to 676 . However, these findings were inconsistent with the results of certain studies. ${ }^{24} \mathrm{~A}$ study conducted by Guo et al reported a statistically significant association with gender, diagnosis, co morbidities and race. ${ }^{22}$ Similarly, our findings showed a statistically significant association with variables such as age, gender, diagnosis, comorbidities and the total number of drugs administered. As ours was a study focusing on patients of the Indian origin, race wasn't a significant factor.

There were potential limitations in this study. The actual effects of the identified pDDIs were not evaluated. Further studies are needed to identify actual clinical consequences of these interactions. The enrolled patients were not continuously evaluated during their entire hospital stay. Irregular follow-up might lead to inconsistency in result. Micromedex $^{(\mathrm{TM})}$ was used for screening of pDDIs in this study while multiple screening resources were used by other researchers. The study was generalized as it was conducted in a tertiary care hospital. Hence, only the pDDIs of the specific drug prescription pattern is determined. Interactions of herbal and topical medications were not evaluated in the study.

\section{Future direction}

Future studies should consider the use of larger sample size. Assessment of ADR's due to DDI's in patients can also be evaluated. Standard treatment guidelines should be made considering most prevalent DDI's, which will reduce 
incidence of ADR's in psychiatric patients. As the number of uses of psychotropic medications is on a rise, the pharmacist's role too needs to be extensive. The pharmacists can serve as an excellent source to assist physicians in their prescribing and counselling patients about psychotropic medications. Documentation of DDI's in specific population is of great importance, which needs a strong nationwide DDI reporting structure.

\section{CONCLUSION}

A high prevalence of pDDIs totalling to 2900 was recorded in our study with an average of 7.33 interactions per patient. Most of the interactions were of major (56.52\%) and moderate severity (39.07) followed by contraindicated (2.55) and minor (1.83). A significant association of the pDDIs with variables such as age, gender, diagnosis and total number of drugs used was also identified. A majority of the population affected by the identified pDDIs belonged to the adult age group (41-50) and of the male gender. Schizophrenia was found to be the common diagnosis among the enrolled patients (225). Upon theoretical analysis a high number of the clinical outcomes were found to have an effect on the cardiovascular system (41.01\%). Trihexyphenidyl, haloperidol, lorazepam, promethazine, divalproex, olanzapine and risperidone were the commonly used antipsychotics and trihexyphenidyl with haloperidol (10.33\%), trihexyphenidyl with promethazine trihexyphenidyl $(9.09 \%)$ with divalproex $(8.74 \%)$, trihexyphenidyl with divalproex $(8.56 \%)$ were the most prevalent interactions seen in our study. More studies are required to explore the overall pattern of DDIs in psychiatric patients along with their levels and correlation with different risk factors. Careful monitoring and documentation are necessary to prevent further complication thereby improving the therapeutic outcome.

\section{ACKNOWLEDGEMENTS}

The author would like to the individuals who extended their valuable assistance for the preparation and completion of this study in one way or other. The patients who agreed to be a part of our study were indeed our biggest asset. Their easy cooperation and dedication were a huge encouragement to step up our work.

\section{Funding: No funding sources}

Conflict of interest: None declared

Ethical approval: The study was approved by the Institutional Ethics Committee of Bharati Medical College, Dhankawadi, Pune, BVDUMC/IEC/83 (Annexure III).

\section{REFERENCES}

1. Stein D, Phillips K, Bolton D, Fulford KWM. Sadler J, Kendler K. What is a Mental/Psychiatric Disorder? From DSM-IV to DSM-V. Available at:
https://www.ncbi.nlm.nih.gov/pmc/articles/PMC310 1504/. Accessed on 2017, November $20^{\text {th }}$.

2. World Health Organization. Mental Disorders. Available http://www.who.int/mediacentre/factsheets/fs396/en/ . Accessed on 2017, November $20^{\text {th }}$.

3. Regier DA, Kuhl EA, Kupfer DJ. Classification and criteria changes. The DSM-5. 1) American Psychiatric Association, Division of Research, Arlington, VA, USA; 2) Department of Psychiatry, University of Pittsburgh Medical Center, Pittsburgh, PA, USA. 2020;94.

4. National Mental Health Survey of India, 2015-2016 Prevalence, Patterns and Outcomes, Supported by Ministry of Health and Family Welfare, Government of India, and Implemented by National institute of Mental Health and Neurosciences (NIMHANS). Bengaluru: In Collaboration with Partner Institutions. 2015-2016.

5. Math SB, Chandrashekar CR, BhugraD. Psychiatric epidemiology in India. Indian J Med Res 2007; 126:183-192.

6. McGrath J, Saha S, Chant D, Welham J. Schizophrenia: a concise overview of incidence, prevalence, and mortality. 2008;30:67-76.

7. Math S, Srinivasaraju R. Indian Psychiatric epidemiological studies: Learning from the past. Indian J Psychiatry. 2010;52(7):95.

8. Shah N, Grover S, Rao G P. Clinical Practice Guidelines for Management of Bipolar Disorder. Indian J Psychiatry. 2017;59(S1):51-66.

9. Felker B, Yazel JJ, Short D. Mortality and medical comorbidity among psychiatric patients: A review. Psychiatr Serv. 1996;47:1356.

10. Merikangas K, Pato M. Recent Developments in the Epidemiology of Bipolar Disorder in Adults and Children: Magnitude, Correlates, and Future Directions. Clin Psychol Sci Practice. 2009;16(2):121-33.

11. Gureje O, Lasebikan VO, Kola L, Makanjuola VA. Lifetime and 12-month prevalence of mental disorders in the Nigerian Survey of Mental Health and Well-Being. Br J Psychiatry. 2006;188:465-71.

12. Grant BF, Stinson FS, Hasin DS. Prevalence, correlates, and comorbidity of bipolar I disorder and axis I and II disorders: results from the National Epidemiologic Survey on Alcohol and Related Conditions. J Clin Psychiatry. 2005;66(10):1205-15.

13. Oxford University Press ANZ. Available at: http://lib.oup.com.au/he/health/samples/edward_psyc hopharmacology_sample.pdf. Accessed on 2017, November $13^{\text {th }}$.

14. Malone DC, Abarca J, Hansten PD. Identification of serious drug-drug interactions: results of the partnership to prevent drug-drug interactions. J Am Pharm Assoc. 2004;44(2):142-51.

15. Johnell K, Klarin I. The relationship between number of drugs and potential drug-drug interactions in the elderly: a study of over 600,000 elderly patients from 
the Swedish Prescribed Drug Register. Drug Saf. 2007;30:911-18.

16. Essock SM, Schooler NR, Stroup TS. Effectiveness of Switching From Antipsychotic Polypharmacy to Monotherapy. Am J Psychiatry. 2011;168:702-8.

17. Sandson NB, Armstrong SC, Cozza KL. An Overview of Psychotropic Drug-Drug Interactions. Psychosomatics. 2005;46(5):464-94.

18. Katon W, Cantrell C, Sokol MC, Chiao E, Gdovin JM. Impact of antidepressant drug adherence on comorbid medication use and resource utilization. Arch Intern Med. 2005;165:2497-503.

19. Demler TL. Psychiatric drug-drug interactions. US Pharmacist. 2012;39:16-9.

20. Psychotropic Drug Interactions. Graylands Hospital Drug Bulletin. 2006;14(2):1323-251.

21. Thomas M, Boggs AA, DiPaula B, Siddiqi S. Adverse drug reactions in hospitalized psychiatric patients. Ann Pharmacother. 2010;44:819-25.

22. Guo JJ, Wu J, Kelton CM, Jing Y, Fan H, Keck PE et al. Exposure to potentially dangerous drug-drug interactions involving antipsychotics. Psychiatr Serv. 2012;63(11):1080-8.

23. McDonnell PJ, Jacobs MR. Hospital admissions resulting from preventable adverse drug reactions. Ann Pharmacother. 2002;36:1331-6.

24. Ismail M, Iqbal Z, Khattak MB. Potential Drug-Drug Interactions in Psychiatric Ward of a Tertiary Care Hospital: Prevalence, Levels and Association with Risk Factors. Trop J Pharm Res. 2012;11(2):289-96.

25. Mangoni AA, Jackson SH. Age-related changes in pharmacokinetics and pharmacodynamics: basic principles and practical applications. $\mathrm{Br} \mathrm{J}$ Clin Pharmacol. 2004;57:6-14.

26. Ho YF, Huang SH, Lin HN. Detecting drug-drug interactions in medication profiles of psychiatric inpatients: a two-stage approach. J Formos Med Assoc. 2002;101;4.

27. Lima RE, De Bortoli Cassiani SH. Potential drug interactions in intensive care patients at a teaching hospital. Rev Lat Am Enfermagem. 2009;17:222-7.

28. Lucca JM, Ramesh M, Ram D, Kishor M. Incidence and predictors of adverse drug reactions caused by drug-drug interactions in psychiatric patients: An empirical study. 201619:1.
29. Ramadan MI, Steven FW, Sheldon HP. Preventing drug drug interactions with mood stabilizers. 2005;4:26-40.

30. Bucşa C, Farcaş A, Cazacu I, Leucuta D, Achimas-Cadariu A, Mogosan $\mathrm{C}$ et al. How many potential drug-drug interactions cause adverse drug reactions in hospitalized patients? Eur J Intern Med. 2013;24:27-33.

31. Sample size calculator. Raosoft R. Available at: http://www.raosoft.com/samplesize.html. Accessed on 2018, April $20^{\text {th }}$.

32. IBM Micromedex ${ }^{\mathrm{TM}}$. Available at: https://www.micromedexsolutions.com/home/dispatc h/ssl/true. Accessed on 2018, January $12^{\text {th }}$.

33. Mezgebe HB, Seid K. Prevalence of potential drugdrug interactions among psychiatric patients in Ayder referral hospital, Mekelle, Tigray, Ethiopia. J Sci Innovative Res. 2015;4(2):71-5.

34. Jain T, Bhandari A, Ram V, Parakh M, Wal P, Nagappa AN. Drug interactions and adverse drug reactions in hospitalized psychiatric patients: A critical element in providing safe medication use. German J Psychiatry. 2011;14:26-34.

35. Sengul M, Karadag F, Sengul C, Karakulah K, Kalkanci O, Herken H. Risk of Psychotropic Drug Interactions in Real World Settings: a Pilot Study in Patients with Schizophrenia and Schizoaffective Disorder. Klinik Psikofarmakoloji Bülteni-Bulletin of Clin Psychopharmacol. 2014;24(3):235-47.

36. Kluwer W. Acsessing Clinical tools. Available at: http://www.wolterskluwercdi.com/factscomparisons-online/user-guide/tools-interactions/. Accessed on 2018, April 20 .

37. Chi-Square test calculator. Math is fun advanced. Available at: https://www.mathsisfun.com/data/chisquare-calculator.html. Accessed on 2018, April $20^{\text {th }}$.

Cite this article as: Jacob A, Thomas CS, Deore A, Deshpande P. Appraisal and discernment of prevalent drug-drug interactions in patients with psychiatric disorders. Int J Basic Clin Pharmacol 2021;10:1005-15. 